\title{
A Confirmatory Factor Analysis of the Wong and Law Emotional Intelligence Scale in a Sample of International College Students
}

\author{
Kok-Mun Ng • Chuang Wang • Carlos P. Zalaquett • \\ Nancy Bodenhorn
}

Published online: 7 June 2008

(C) Springer Science + Business Media, LLC 2008

Due to an error in production, the wrong version of "A Confirmatory Factor Analysis of the Wong and Law Emotional Intelligence Scale in a Sample of International College Students" (Volume 29, December 2007, pp. 173-185, DOI: 10.1007/s10447-007-9037-6) was erroneously published online and in print. The correct version of the article follows in its entirety. Springer regrets the error.

The online version of the original can be found at doi:10.1007/s10447-007-9037-6

K.-M. Ng $(\bowtie) \cdot$ C. Wang $\cdot$ C. P. Zalaquett $\cdot$ N. Bodenhorn

Counseling, University of North Carolina at Charlotte, 9201 University City Blvd., Charlotte, NC 28223, USA

e-mail: kokmunng@uncc.edu 


\title{
A Confirmatory Factor Analysis of the Wong and Law Emotional Intelligence Scale in a Sample of International College Students
}

\author{
Kok-Mun Ng • Chuang Wang • Carlos P. Zalaquett • \\ Nancy Bodenhorn
}

Published online: 16 October 2007

(C) Springer Science + Business Media, LLC 2007

\begin{abstract}
There is the need for a reliable and valid measure to facilitate emotional intelligence (EI) research on international college students (ICSs). The present study examined the factorial invariance of the Wong and Law Emotional Intelligence Scale (WLEIS), a trait EI measure, in a sample of 628 ICSs. A web-based survey was developed to facilitate data collection across the country. Results of a confirmatory factor analysis support the factorial invariance of the WLEIS in ICSs. Reliabilities and scale correlations further supported the psychometric properties of the measure for international students. Additional findings indicate possible country-of-origin difference on trait EI among different national groups.
\end{abstract}

Keywords Assessment - Emotional intelligence $\cdot$ Factor analysis ·

International college students

\section{Introduction}

The concept of emotional intelligence (EI) has gained much attention from researchers and practitioners across disciplines since its introduction in 1990 by Salovey and Mayer. Scores of studies have provided empirical evidence to support the concept's usefulness in understanding a wide range of human functioning (e.g., Austin et al. 2005; Brackett et al. 2004; Ciarrochi et al. 2002; Extremera and Fernandez-Berrocal 2006; Law et al. 2004; Slaski and Cartwright 2002). Studies on EI in recent years have also started to emerge from non-Western countries (e.g., Wong and Law 2002; Fukunishi et al. 2001; Bhattacharya et al. 2004). Researchers have also begun to investigate the relationship between culture/ethnicity and EI (e.g., Bhattacharya et al. 2004; Parker et al. 2005; Shipper et al. 2003; Van Rooy et al. 2005). However, little literature has examined EI in international college students (ICSs), who

K.-M. Ng $(\bowtie) \cdot$ C. Wang $\cdot$ C. P. Zalaquett $\cdot$ N. Bodenhorn Counseling, University of North Carolina at Charlotte, 9201 University City Blvd, Charlotte, NC 28223, USA

e-mail: kokmunng@uncc.edu 
have been found to experience socio-emotional functioning and academic related issues of kinds both common to college students as well as unique to them (Pedersen 1991; Poyrazli et al. 2001). There is also a lack of EI measures that have been validated for use to study this student population.

This paper reports a validation study of the Wong and Law Emotional Intelligence Scale (WLEIS) (Wong and Law 2002) on a large sample of ICSs in the US. The WLEIS was originally developed based on Hong Kong samples and, to date, it has not been further validated on other populations. To this point, the use of the measure is limited to studies conducted by its original developers (Wong and Law 2002; Law et al. 2004) and two doctoral dissertations (Cintron 2005; Sitter 2005). The measure will be described in greater detail in the method section. Though the measure was noted to capture a limited portion of the trait EI domains (Tett et al. 2005), it seems to be a promising research tool because of its brevity, compared to existing trait EI measures, and its demonstrated psychometric properties. Additional validation studies on different populations will help to verify the measure's utility beyond that in the Hong Kong setting.

Professional standards for educational and psychological testing further underscore the significance of the present study. Such standards require that the validation process of a measure be ongoing with continuing efforts to establish the usefulness of the measure for specific populations and purposes (American Educational Research Association, American Psychological Association, and National Council on Measurement in Education 1999). Therefore, the main focus of the present study was to examine the replicability of the factor structure of the English version of the WLEIS as reported by its developers in a sample of ICSs.

\section{International College Students}

Despite the slight drop in 2003/04 and 2004/05 enrollments, international student numbers in US higher education have grown dramatically since the 1950s (Institute of International Education (IIE) 2005). In the 2004/05 academic year, there were 565,039 international students, constituting $4.0 \%$ of overall student enrollment. The growth of ICSs has resulted in such students becoming the focus of many studies.

The needs of international students are considerable because of the high levels of adjustment-related stressors they encounter (Chen 1999). These students face common developmental problems related to living away from their families for the first time (Pedersen 1991). They also encounter problems unique to their status as international students. Chen (1999) reviewed the literature and listed the following common stressors among international students' experiences: (a) second language anxiety, (b) educational stressors (i.e., performance expectations, system adjustment, and test-taking anxiety), and (c) social stressors (i.e., culture shock, social isolation and alienation, financial concerns, and racial discrimination and prejudice). In addition, these students also have to contend with legal issues; for example, facing possible deportation if they fail to maintain the required number of credit hours, even if they find their course load too difficult to manage (Collingridge 1999). International students in other Western countries besides the US have been found to face similar issues (Robertson et al. 2000). Though there is considerable literature on the needs and experiences of international students, Yoon and Portman's (2004) review of the literature revealed that there is a scarcity of assessment instruments developed or validated for the international student population. Findings on socioemotional functioning of ICSs based on measures lacking demonstrated psychometric properties can result in misleading interpretations (Yoon and Portman 2004). 
Parker et al. (2005) cautioned that special care is needed when using the EI construct in different cultures because culture can influence the experience and expression of emotions. This caution was supported by findings in the literature that showed cultural differences on experience and expression of emotions (e.g., Scollon et al. 2004). This is particularly important when studying international students in that typically they are immensely heterogeneous because they originate from diverse national and cultural backgrounds. The lack of a valid EI instrument interferes with research on the relationships between EI and the social and emotional functioning of ICSs. The present study represents an attempt to address the need for validated measures for use on international students, particularly in the study of EI.

\section{Emotional Intelligence}

EI, which refers to the ability of people to deal with their emotions, is rooted in the concept of "social intelligence" which was first proposed by Thorndike (1920) and later explicated by Gardner (1993) to include interpersonal and intrapersonal intelligences. Salovey and Mayer (1990) were the first to propose a formal conceptualization of EI. Currently, the literature presents two distinct views of EI: ability and disposition. From the ability approach, EI is perceived as a capacity to engage in valued behavior and entails a degree of mutability. Mayer et al. (1999) defined this as:

an ability to recognize the meanings of emotions and their relationships, and to reason and problem-solve on the basis of them. Emotional intelligence is involved in the capacity to perceive emotions, assimilate emotion-related feelings, understand the information of those emotions, and manage them. (p. 267)

Ability EI is assessed using performance measures; for example, the Mayer-Salovey-Caruso Emotional Intelligence Test (Mayer et al. 2002).

From the disposition approach, EI is a relatively stable tendency amenable to selfdescription (Tett et al. 2005). In terms of this approach, Petrides and Furnham (2003) defined EI as "a constellation of behavioral dispositions and self-perceptions concerning one's ability to recognize, process, and utilize emotion-laden information" (p. 278). Petrides and Furnham proposed the term "trait emotional intelligence" (trait EI) to distinguish the disposition approach from the ability approach. The literature considers these two approaches as complementary (Tett et al. 2005). Trait EI is assessed via self-report measures; for example, the WLEIS.

Davies et al.'s (1998) review of the EI literature led them to explicate the dimensions of EI as follows:

1. Appraisal and expression of emotion in oneself. This relates to a person's ability to become aware both of their mood and their thoughts concerning that mood.

2. Appraisal and recognition of emotions in others. This relates to a person's ability to perceive and understand the emotions of others.

3. Regulation of emotion in oneself and others. This relates to a person's ability to regulate his or her emotions; that is, to monitor, evaluate, and act to change one's mood. Regulation of emotion also includes the ability to change the affective reactions of others.

4. Use of emotion to facilitate performance. This relates to the ability of the person to utilize his or her emotions by directing them toward constructive endeavors and performance. 
Conceptually, individuals with higher levels of EI are able to make use of their emotion regulation mechanisms effectively to create positive emotions as well as to promote emotional and intellectual growth (Wong and Law 2002). Wong and Law developed their trait EI scales involving four domains in accordance with the schematic of EI of Davies et al., which reflected Salovey and Mayer's (1990) conceptualization. However, it should be noted that several extant trait EI measures include a greater number of domains. For example, Tett et al.'s (2005) trait EI measure consists of 10 facets as proposed by Salovey and Mayer (1990) and Petrides and Furnham's (2003) has 15 facets. Detailed discussion of the various trait EI models is outside the scope of the present study. Research on EI is young and efforts to clarify the theories and measures of EI are still underway (Brackett and Geher 2006).

Despite the relatively short history of EI, empirical evidence supporting its usefulness in understanding a wide range of human functioning is being gathered (Tsaousis and Nikolaou 2005). Among the studies that involved college students, EI has been found to positively correlate to college academic performance (Parker et al. 2004; Schutte et al. 1998; Van der Zee et al. 2002), social network size and quality (Austin et al. 2005; Van der Zee et al. 2002). EI significantly accounted for quality of social interactions after controlling for personality variables (Lopes et al. 2004). EI was also found to moderate the relationship between stress and mental health among university students (Ciarrochi et al. 2002; Extremera and Fernandez-Berrocal 2006). Brackett et al. (2004) found EI to be associated with maladjustment and negative behaviors for college-aged males. Extremera and Fernandez-Berrocal's study (2006) on 184 university students found that mood repair, a trait EI factor measured by the Trait Meta-Mood Scale, positively predicted $4 \%$ of students' general health.

Despite many EI studies, very limited research has focused on or included ICSs. Hence, generalizability of the findings to this student population needs further empirical investigation. To the best of our knowledge, Rozell et al.'s (2002) EI study is the only one in peer-reviewed literature that involved ICSs in the US. They reported that international students rated lower on the EI measure as compared to domestic students. Rozell et al. noted that the observed differences might have been due to cultural differences and further research needed to be conducted to examine the causes of these cultural differences in terms of the EI measures. They asserted, "However, if one assumes that cultural test score bias is not the cause of the cultural differences, this finding indicates that individuals [international students] who are trying to succeed in business may discover that their opportunities are limited by their EI" (p. 286).

But, findings in that study were confounded by several issues. First, the study was based on Goleman's (1995) model of EI, which has been questioned in terms of its validity because of its expansiveness (e.g., Matthews et al. 2002). The model includes a vast array of skills that do not have scientific support for inclusion as domains of EI (Pfeiffer 2001; Sternberg 2001). Second, the study was limited to students from business majors. Of the 295 participants, there were only 76 international students in the study. The authors did not provide additional background information on these international students. However, international students are a very heterogeneous group of people. The authors did not note the limitations related to within-group difference when interpreting their findings on international students' EI.

Furthermore, Rozell et al. (2002) had not specifically examined the validity of the measure on international students in their study, though they did examine the validity of the measure on the whole research sample. Therefore, the findings of Rozell et al. may lead to a premature conclusion that international students are deficient in EI in comparison to 
domestic students. Further research is needed to investigate what factors underlie the score differential between the student groups. Such cross-cultural comparative studies require research instruments that have demonstrated comparable psychometric properties on the groups being studied.

In view of the above, the purpose of the present study was to investigate the applicability of the WLEIS to ICSs by examining the measure's factor invariance in a sample of ICSs. It is acknowledged that international students are highly heterogeneous. However, due to subgroup sample-size limitation, the present study was not able to examine the factor structure of the WLEIS in each subgroup of ICSs. Therefore, the focus was limited to ICSs in general.

\section{Materials and Methods}

The authors developed an online survey to conduct the present study. Internet research poses some methodology concerns; for example, potential low response rates, selfselectivity of Internet users, technological issues with the deployment of the research tool, concerns over Internet security, multiple completions by the same individual, and uninvited respondents entering the sample (Heerwegh and Loosveldt 2002; Sills and Song 2002). Because of this, the authors had to carefully screen the data to remove 'suspicious' entries.

Despite the problems associated with web-based research, it has gained popularity in recent years. Such studies include research across a wide spectrum of subject matter (Sills and Song 2002). Web-based studies have been found to be a sensible means of achieving meaningful results for populations that regularly use the Internet in their daily lives (Sills and Song 2002), and we contend that ICSs are a good example in this regard. Notwithstanding the problems associated with web-based surveys, online procedures allowed the researchers to reach their target population more economically and extensively. We were able to recruit a large number of participants from campuses across the US who originated from many different countries, instead of only relying on students from limited locations.

\section{Participants}

We received a total of 691 response sets online. These responses came from ICSs residing in Washington, D.C. and 28 other states (i.e., AK, AL, AR, AZ, CA, CO, KY, FL, GA, ID, IL, IN, LA, MA, MD, MI, MN, MO, NC, ND, NY, OK, OH, PA, SD, WA, TX, and VA). Based on careful screening of the data, cases were deleted if they: (a) contained too many incomplete survey items that prevented meaningful analysis; (b) comprised identical item response ratings within and across instruments; and (c) were identical to another response set, indicating potential multiple submissions. This resulted in a final total of 628 usable response sets (344 females, 281 males, and 3 unidentified). The mean age for the sample was 26.2 years $(S D=5.1)$. Twenty four participants did not report their age. There were nine non-degree students, 187 undergraduate students, and 341 graduate students.

Participants represented 92 countries from Africa, Asia, Europe, Latin America, North America, and Oceania. The nine most represented countries were India $(n=109)$, China $(n=$ $65)$, Korea $(n=46)$, Japan $(n=35)$, Taiwan $(n=29)$, Canada $(n=19)$, Malaysia $(n=17)$, Brazil $(n=15)$, and Germany $(n=15)$. National make-up of the participants to a large extent appears to match the list of leading countries of origin of international students. The Institute of International Education (2005) reported the following ranking of place of origin 
for international students in 2004/2005: 1st, India; 2nd, China; 3rd, Korea; 4th, Japan; 5th, Canada; 6th, Taiwan; 9th, Germany; 14th, Brazil; and 20th, Malaysia.

\section{Measure}

\section{Wong and Law Emotional Intelligence Scale}

Wong and Law (2002) developed the measure based on Davis et al.'s (1998) summary of EI in the literature, which consists of four domains. These domains form the four subscales of the measure. Each subscale consists of four items. The samples on which Wong and Law based their development of the measure were Hong Kong undergraduate and graduate students and working adults.

Wong and Law (2002) used three groups of independent samples to develop the items and test their psychometric properties. The first group consisted of two samples: Sample One $(n=120)$ comprised managers and students who assisted in item generation and Sample Two $(n=189)$ was made up of undergraduate students who provided quantitative evidence in regard to item selection. The second group comprised "two cross-validated samples $(n=$ 72 and $n=146$ ) who provided quantitative evidence to confirm the factorial structure of the four EI dimensions and their relationships with the external criterion variables" (p. 252). The third group consisted of two other samples (i.e., 110 undergraduate students and 116 non-teaching employees in a university) used to test the various types of validity. Wong and Law did not provide additional demographic details of their samples.

Wong and Law (2002) selected a total of 16 items, four per dimension, because they had the largest factor loadings (average loading=0.80) on the dimensions. The four-factor solution explained $71.5 \%$ of the variance. The items on the measure are self-rated on a 7 point Likert-type scale $(1=$ totally disagree to $7=$ totally agree $)$. The four scales of measure are: Self-Emotion Appraisal (SEA), Others' Emotion Appraisal (OEA), Use of Emotion (UOE), and Regulation of Emotion (ROE). All items are positively keyed; and this poses as a weakness of the measure. A sample item from SEA is "I have a good sense of why I have certain feelings most of the time." A sample item from OEA is "I always know my friend's emotions from their behavior." "I always set goals for myself and then try my best to achieve them" and "I have good control of my own emotions" are items from UOE and ROE respectively.

The interscale correlations were mild to moderate in one sample $(r=0.13-0.42)$ and higher in another sample $(r=0.60-0.76)$ (Wong and Law 2002). The ranges of coefficient alphas for the scales reported in various studies in Wong and Law's article were 0.86-0.92 for SEA, $0.82-0.93$ for OEA, $0.84-0.88$ for UOE, and $0.71-0.91$ for ROE. No test-retest reliability was reported.

Wong and Law (2002) tested the psychometric properties of the WLEIS with three groups of independent samples of undergraduate students. The convergent, incremental and discriminate validities of the measure were tested with additional independent samples. EI as measured by the WLEIS correlated significantly with job performance $(r=0.21)$ and job satisfaction $(r=0.40)$. The scales were also negatively correlated with powerlessness $(r=-0.13$ to -0.39$)$ and positively correlated with life satisfaction $(r=0.16-0.46)$ as hypothesized. The WLEIS was found to correlate moderately with EQ-i, a trait EI measure developed by Bar-On (2000) $(r=0.63)$. The scales had minimal correlations with IQ estimates $(r=-0.19-$ 0.06). Incremental validity of the WLEIS was demonstrated by using it to predict life satisfaction in a hierarchical regression by controlling for the Big Five personality dimensions (Extraversion; Agreeableness; Conscientiousness; Neuroticism; Openness) 
which shared a statistically significant portion of the variances of life satisfaction $\left(R^{2}=\right.$ $0.466, p<0.01)$. The WLEIS significantly explained the additional portion of the variances of life satisfaction $\left(R^{2}=0.077, p<0.01\right)$. Using factor analysis, Wong and Law examined the relationships among the four EI scales and the Big Five personality indicators and found that: (a) UOE and Neuroticism loaded together, (b) ROE and Conscientiousness loaded together, (c) SEA and OEA did not cross-load with the Big Five factors. Law et al.'s (2004) findings further supported the construct and criterion validity of the WLEIS.

\section{Procedure}

\section{Sampling}

The data on the WLEIS were collected as part of an online survey developed to study the social-emotional functioning of ICSs attending American universities. E-mail invitations were sent to international student advisors from the 20 universities with the highest enrollment of international students and from two other universities randomly selected in each state. The e-invite was also sent to those whose e-mails were found as contacts on the Association of International Educators' website. The researchers asked the advisors to forward the participation request to students in their universities. We also sent requests for participation to international students we know and asked them to forward the request to other international students they knew. The e-invite included the hyperlink to the website of the research. Students had a choice to enter into a sweepstakes to win one of $40 \$ 40$ awards upon completion of the survey. Participation was anonymous; however, contact information of participants who entered the sweepstakes was gathered separately from their survey response in order to facilitate the drawing process.

\section{Data Analysis}

A confirmatory factor analysis (CFA) was conducted with LISREL 8.7 to test the fit of the four-factor model to the data. We used multiple criteria to assess the goodness-of-fit of data to the model as recommended in the literature (Tabachnick and Fidell 1996). After confirmation of the factorial validity of the WLEIS, we examined the scale means and variances and internal consistencies. It was also decided to include in the report descriptive statistics of the measure of subgroups according to country of origin with sample sizes greater than 25 .

\section{Results}

Confirmatory Factor Analysis

Test of fitness of the WLEIS' four-factor structure for the whole sample yielded a $\chi^{2}$ value of 479.03 with a degree of freedom of 98 , which suggests that the hypothesized model is not entirely adequate. Nevertheless, finding a well-fitting model of which the $\chi^{2}$ value approximates the degrees of freedom is quite unrealistic in most empirical research (Byrne 1998). Thus, a large $\chi^{2}$ value only indicates that there is a need to modify the model in order to better fit the data. This is not surprising because each $\chi^{2}$ value obtained in the CFA of WLEIS in previous studies also significantly exceeded its degrees of freedom (Law et al. 2004; Wong and Law 2002). 
Root Mean Square Error of Approximation (RMSEA) value for our sample was 0.078 and the $90 \%$ confidence interval ranged from 0.071 to 0.085 . The narrow confidence interval, a range of 0.014 , suggested good precision of the RMSEA value, and 0.078 indicated acceptable fitness. Models whose RMSEA is 0.10 or more have poor fit. Both goodness-of-fit index $(\mathrm{GIF}=0.91)$ and adjusted goodness-of-fit (AGFI=0.88) indices were close to one, suggesting that the four-factor model fits the data quite well (Hu and Bentler 1995).

Normed Fit Index (NFI), Non-Normed Fit Index (NNFI), and Comparative Fit Index (CFI) are often used to evaluate the gain in improved fit from the independence model to the hypothesized model and values of NFI greater than 0.90 and NNFI and CFI greater than 0.95 indicate good fit (Hu and Bentler 1999). In our sample, the NFI, NNFI, and CFI values all equalled to 0.97 , suggesting good incremental fit indices.

Standardized Root Mean Square Residual (SRMR) represents the average standardized residual value derived from the fitting of the correlation matrix for the hypothesized model to that of the sample data. A value less than 0.05 suggests a well-fitting model (Byrne 1998). The value of 0.044 for our sample represents the average discrepancy between the sample observed and hypothesized correlation matrices and can be interpreted as the model explains the correlations to within an average error of 0.044 (see $\mathrm{Hu}$ and Bentler 1995).

Using the joint criteria suggested by $\mathrm{Hu}$ and Bentler (1999), that NNFI and CFI were both greater than or equal to 0.96 and SRMR was less than or equal to 0.09 , the four-factor structure of the WLEIS was found to have adequate fit to the data for the international student sample. As such, we proceeded to further examine the other psychometric properties of the WLEIS in our study.

\section{Item and Scale Analyses}

Cronbach's coefficient alphas for the four dimensions of WLEIS were 0.84 for SEA, 0.84 for OEA, 0.85 for UOE, and 0.87 for ROE. Alpha for the entire WLEIS was 0.91. These values are comparable to those reported by Wong and Law (2002) and Law et al. (2004). The distribution of each item was slightly negatively skewed with means ranging from 4.81 to 5.99 and standard deviations ranging from 1.07 to 1.60 . These item descriptive statistics seem somewhat higher that those reported in Wong and Law's (2002) study (Ms=4.25-4.94 and $S D s=1.20-1.43$ ).

The correlations among the four WLEIS dimensions were moderate to moderately high and in expected directions (see Table I). These were somewhat similar to those found in one of the samples in Law et al. (2004) $(r=0.33-0.54)$. Though the correlations between SEA and OEA and SEA and ROE were moderate to high in our sample, similar magnitudes were also found in Study 2 of Wong and Law (2002, p. 264). However, most

Table I Descriptive Statistics and Correlations Among the Four Dimensions of WLEIS

\begin{tabular}{llllll}
\hline & $\bar{X}$ & $S D$ & SEA & OEA & UOE \\
\hline SEA & 5.48 & 1.19 & & & \\
OEA & 5.42 & 1.17 & $0.69^{*}$ & $0.50^{*}$ & \\
UOE & 5.70 & 1.25 & $0.55^{*}$ & $0.44^{*}$ & $0.49^{*}$ \\
ROE & 5.05 & 1.44 & $0.73^{*}$ & & \\
\hline
\end{tabular}

$\mathrm{SEA}=$ Self-Emotion Appraisal, OEA = Others' Emotion Appraisal, UOE $=$ Use of Emotion, and ROE $=$ Regulation of Emotion. WLEIS $=$ Wong and Law Emotional Intelligence Scale. $n=628 .{ }^{*} p<0.01$. 
interscale correlations reported by Wong and Law in three other samples were mild to moderate.

\section{Scale Means and Standard Deviations}

Means and standard deviations of the four EI subscales are presented in Table I. Means in our sample ranged from 5.05 to 5.70 and standard deviations ranged from 1.17 to 1.44. These descriptive statistics were higher than those reported by Wong and Law (2002) (Ms= $4.50-4.70 ; S D \mathrm{~s}=0.91-0.97)$ as well as those by Law et al. $(2004)(M \mathrm{~s}=3.78-4.15 ; S D \mathrm{~s}=$ $0.96-1.05$; and $M \mathrm{~s}=4.27-4.84 ; S D \mathrm{~s}=0.99-1.15)$. The apparent means differences between studies might be related to characteristics of the samples or sampling bias. Wong and Law did not provide any normative data on the measure. Hence, the apparent differences in descriptive statistics cannot be interpreted meaningfully at this time. Further study is needed to clarify the difference.

The present study represents the first to examine the WLEIS on a large sample of ICSs. Though there was not a large enough sample size for any of the subgroups of students per country to perform CFA to investigate the factor structure of the measure, it was decided to report the means and standard deviations of the four subscales of students from five countries (i.e., India, China, Taiwan, Korea, and Japan) that had sample sizes greater than 25. Table II presents the results. This information may provide a point of reference for future studies that focus on examining race/ethnicity difference on EI.

Students originally from these five countries $(n=283)$ represented $45.06 \%$ of the whole sample and 29 states of the US. Among them, there were two non-degree students, 58 undergraduate students, and 223 graduate students (including four postdoctoral fellows). One participant did not indicate academic status. Cronbach's alphas (see Table II) and interscale correlations were rerun for the reliability and validity of the four WLEIS dimensions for each group (country) of students.

Table II Means and Standard Deviations of WLEIS Dimensions for Five Countries

\begin{tabular}{|c|c|c|c|c|c|}
\hline Dimension & Country & $n$ & $\bar{X}$ & $S D$ & $\alpha$ \\
\hline \multirow[t]{5}{*}{ SEA } & China & 65 & 5.44 & 0.99 & 0.82 \\
\hline & Taiwan & 29 & 5.49 & 1.21 & 0.84 \\
\hline & India & 109 & 5.63 & 0.90 & 0.82 \\
\hline & Japan & 35 & 5.71 & 0.96 & 0.84 \\
\hline & Korea & 46 & 5.14 & 0.91 & 0.80 \\
\hline \multirow[t]{5}{*}{ OEA } & China & 65 & 5.45 & 0.82 & 0.79 \\
\hline & Taiwan & 29 & 5.42 & 1.04 & 0.87 \\
\hline & India & 109 & 5.55 & 0.90 & 0.76 \\
\hline & Japan & 35 & 5.17 & 1.18 & 0.90 \\
\hline & Korea & 46 & 5.05 & 1.02 & 0.89 \\
\hline \multirow[t]{5}{*}{ UOE } & China & 65 & 5.53 & 0.94 & 0.77 \\
\hline & Taiwan & 29 & 5.70 & 1.08 & 0.88 \\
\hline & India & 109 & 5.82 & 1.09 & 0.89 \\
\hline & Japan & 35 & 5.54 & 0.85 & 0.76 \\
\hline & Korea & 46 & 5.46 & 0.93 & 0.84 \\
\hline \multirow[t]{5}{*}{ ROE } & China & 65 & 4.88 & 1.28 & 0.91 \\
\hline & Taiwan & 29 & 5.02 & 1.24 & 0.90 \\
\hline & India & 109 & 5.46 & 1.10 & 0.81 \\
\hline & Japan & 35 & 5.02 & 1.16 & 0.88 \\
\hline & Korea & 46 & 4.75 & 1.19 & 0.87 \\
\hline
\end{tabular}


MANOVA results indicated that students from these countries differed with respect to the four dimensions of WLEIS (Wilks' $\Lambda=0.87, F(16,843)=2.40, p=0.002, \eta^{2}=0.03$ ). Tests of between-subjects effects revealed that students from these five countries differed for SEA, $F(4,279)=2.75, p=0.029, \eta^{2}=0.04$; OEA, $F(4,279)=2.84, p=0.025, \eta^{2}=0.04$; and ROE, $F(4,279)=4.30, p=0.002, \eta^{2}=0.06$; but not for UOE, $F(4,279)=1.59, p=$ $0.178, \eta^{2}=0.02$.

Post Hoc multiple comparisons with Tukey's HSD test indicated that the Indian students' ROE mean score was statistically significantly higher than that of Chinese ( $p=$ $0.017)$ and Korean students $(p=0.006)$. The $95 \%$ confidence intervals for these differences ranged from 0.06 to 1.10 and from 0.13 to 1.29 , respectively. Indian students' OEA mean score was statistically significantly higher than that of Korean students $(p=0.03)$. The $95 \%$ confidence interval for this difference was between 0.03 and 0.97 .

As for the SEA mean scores, Korean students reported statistically lower levels than Indian students $(p=0.03)$. The 95\% confidence intervals for these differences ranged from -0.95 to -0.02 . Although Japanese students scored higher on SEA $(M=5.71, S D=$ $0.96)$ than Korean students $(M=5.14, S D=0.91)$, this difference was not statistically significant. The $95 \%$ confidence interval of the difference was from -1.17 to 0.02 . All other pairwise comparisons of the four dimensions of WLEIS did not show any statistically significant differences between these countries $(p s>0.05)$.

\section{Discussion}

The main purpose of the study was to examine the factor invariance of the WLEIS in international students. The CFA results represent the first evidence to support the factorial invariance and hence the utility of the WLEIS with ICSs. Coupled with its brevity and current findings on its factorial validity, we recommend the use of the WLEIS to investigate international students' EI and its correlates.

The confirmative findings allowed the researchers to proceed with further examination of other psychometric properties of the measure. The measure and its four subscales showed acceptable levels of reliability with international students. The reliability coefficients were comparable in magnitude to those reported by Wong and associates (Law et al. 2004; Wong and Law 2002). Interscale correlations for the entire sample were in the expected direction and within the range reported by Wong and Law (2002). These correlations further support the measure's content validity.

Studies on international students have tended to overgeneralize results to all international students without recognizing within group differences in this student population (Yoon and Portman 2004). This study is the first to examine ICS's country-of-origin differences on trait EI. The findings indicate that: (a) Indian students reported higher levels of ROE than did both Chinese and Korean students; (b) Indian students also reported higher levels of OEA and SEA than Korean students; and (c) Korean students reported the lowest levels on all four EI dimensions. Though the findings on country of origin difference on EI, an indication of race/ethnicity difference, are at best preliminary, they are in alignment with the literature on ethnic and nationality differences on trait EI (e.g., Ghorbani et al. 2002; Parker et al. 2005; Shipper et al. 2003). The findings also validate the call for attention to group differences when studying ICSs (Yoon and Portman 2004). The present study is limited in its scope and was not able to provide information that might shed light on the group differences on dimensions of EI among international students. We recommend future studies to focus on examining variables that underlie such differences. 
The present study indicates that the WLEIS has acceptable reliability and validity to be used in researching the generalizability of findings regarding trait EI on ICSs in general, though caution should be taken with its usage with Korean students. And, researchers should consider controlling for country-of-origin when analyzing the data. We recommend further studies to investigate reasons leading to Korean students' overall lower EI scores.

Though the study included a large sample of international students across many states in order to ensure a better representativeness compared to many extant studies involving ICSs, and the countries represented among participants appear to closely match the ranking of leading countries of origin of international students, there remains several limitations. First, insufficient sample size for subgroups precluded CFA from being performed on group levels. The CFA findings only support the utility of the WLEIS for international students in general. Additional CFA studies on various national groups should be done to further verify the factorial invariance of the WLEIS.

Second, though utilization of a web-based design allowed for a large sample of participants, it was necessary to eliminate about $9 \%$ of the cases that were deemed unusable. Better measures to prevent multiple submissions and incomplete submissions could have been put in place. Online surveying is still a new research method. Limitations and external threats of validity have yet to be fully understood thus far. Therefore, interpretation of the findings needs to bear in mind the non-traditional sampling procedure used. Replicatory factor analytic study on the WLEIS using traditional sampling procedures should be carried out to verify findings of the present study.

\section{Conclusion}

Notwithstanding the limitations, this study represents the very first in the literature to study the factorial invariance of an EI measure in international students and provides empirical support for its use on this student population. We believe that the size of the sample, the vast geographic spread of participants, and a close match to the list of leading countries of origin for international students compensated the lack of randomization of the sample. We believe the findings extend the utility of the WLEIS in international students in general. The findings also accentuate the need to examine and account for within-group differences when studying this student population in general, and in particular, in regard to EI. This study further validates the professional standards for educational and psychological testing that require the validation process to involve continuing efforts to establish the usefulness of a measure for specific populations and purposes.

Acknowledgement Research reported in this article was funded in part by a grant provided by the Research \& Assessment Corporation for Counselors, Inc., formerly an affiliate of the National Board for Certified counselors.

\section{References}

American Educational Research Association, American Psychological Association, and National Council on Measurement in Education. (1999). Standards for educational and psychological testing. Washington, DC: American Educational Research Association.

Austin, E. J., Saklofske, D. H., \& Egan, V. (2005). Personality, well-being and health correlates of trait emotional intelligence. Personality and Individual Differences, 38, 547-558. 
Bar-On, R. (2000). Emotional and social intelligence: Insights from the Emotional Quotient Inventory. In R. BarOn, \& J. D. A. Parker (Eds.), The handbook of emotional intelligence: Theory, development, assessment, and application at home, school, and in the workplace (pp. 363-388). San Francisco: Jossey-Bass.

Bhattacharya, M., Dutta, A. K., \& Mandal, M. K. (2004). Factor structure of emotional intelligence in India. Psychological Studies, 49, 142-146.

Brackett, M. A., \& Geher, G. (2006). Measuring emotional intelligence: Paradigmatic diversity and common ground. In J. Ciarrochi, J. P. Forgas, \& J. D. Mayer (Eds.), Emotional intelligence in everyday life (2nd ed., pp. 27-50). New York: Psychological Press.

Brackett, M. A., Mayer, J. D., \& Warner, R. M. (2004). Emotional intelligence and its relation to everyday behavior. Personality and Individual Differences, 36, 1387-1402.

Byrne, B. M. (1998). Structural equation modeling with LISREL, PRELIS, and SIMPLIS: Basic concepts, applications, and programming. Mahwah, NJ: Lawrence Erlbaum.

Chen, C. P. (1999). Common stressors among international college students: Research and counseling implications. Journal of College Counseling, 2, 49-65.

Ciarrochi, J., Deane, F. P., \& Anderson, S. (2002). Emotional intelligence moderates the relationship between stress and mental health. Personality and Individual Differences, 32, 197-209.

Cintron, N. E. (2005). The effects of biculturalism, emotional intelligence, and acculturation on motivation to lead of expatriate Latina business leaders and entrepreneurs: An exploratory investigation. Dissertation Abstracts International, 65(7-A), 2778.

Collingridge, D. S. (1999). Suggestions on teaching international students: Advice for psychology instructors. Teaching of Psychology, 26, 126-128.

Davies, M., Stankov, L., \& Roberts, R. D. (1998). Emotional intelligence: In search of an elusive construct. Journal of Personality and Social Psychology, 75, 989-1015.

Extremera, N., \& Fernandez-Berrocal, P. (2006). Emotional intelligence as predictor of mental, social, and physical health in university students. Spanish Journal of Psychology, 9, 45-51.

Fukunishi, I., Wise, T. N., Sheridan, M., Shimai, S., Otake, K., Utsuki, N., et al. (2001). Validity and reliability of the Japanese version of the emotional intelligence scale among college students and psychiatric outpatients. Psychological Reports, 89, 625-632.

Gardner, H. (1993). Multiple intelligences: The theory in practice. New York: Basic Books.

Ghorbani, N., Bing, M. N., Watson, P. J., Davison, H. K., \& Mack, D. A. (2002). Self-reported emotional intelligence: Construct similarity and functional dissimilarity of higher-order processing in Iran and the United States. International Journal of Psychology, 37, 297-308.

Goleman, D. (1995). Emotional intelligence: Why it can matter more than IQ. New York: Bantam.

Heerwegh, D., \& Loosveldt, G. (2002). Web surveys: The effect of controlling survey access using PIN numbers. Social Science Computer Review, 20, 10-21.

Hu, L, \& Bentler, P. M. (1995). Evaluating model fit. In R. H. Hoyle (Ed.), Structural equation modeling: Concepts, issues, and application (pp. 76-99). Thousand Oaks, CA: Sage.

Hu, L., \& Bentler, P. M. (1999). Cutoff criteria for fit indexes in covariance structure analysis: Conventional criteria versus new alternatives. Structural Equation Modeling: A Multidisciplinary Journal, 6, 1-55.

Institute of International Education. (2005). In H. -K. Koh Chin (Ed.), Open doors: Report on international educational exchange. New York: Institute of International Education.

Law, K. S., Wong, C., \& Song, L. J. (2004). The construct and criterion validity of emotional intelligence and its potential utility for management studies. Journal of Applied Psychology, 89, 483-496.

Lopes, P. N., Brackett, M. A., Nezlek, J. B., Schutz. A., Sellin, I., \& Salovey, P. (2004). Emotional intelligence and social interaction. Personality and Social Psychology Bulletin, 30, 641-658.

Matthews, G., Zeidner, M., \& Roberts, R. D. (2002). Emotional intelligence: Science and myth. Cambridge, MA: The MIT Press.

Mayer, J. D., Caruso, D. R., \& Salovey, P. (1999). Emotional intelligence meets traditional standards for an intelligence. Intelligence, 27, 267-298.

Mayer, J. D., Salovey, P., \& Caruso, D. R. (2002). Mayer-Salovey-Caruso Emotional Intelligence Test user's manual. Toronto, Canada: Multi-Health Systems.

Parker, J. D. A., Saklofske, D. H., Shaughnessy, P. A., Huang, S. H. S., Wood, L. M, \& Eastabrook, J. M. (2005). Generalizability of the emotional intelligence construct: A cross-cultural study of North American aboriginal youth. Personality and Individual Differences, 39, 215-227.

Parker, J. D. A., Summerfeldt, L. J., Hogan, M. J., \& Majeski, S. A. (2004). Emotional intelligence and academic success: Examining the transition from high school to university. Personality and Individual Differences, 36, 163-172.

Pedersen, P. (1991). Counseling international students. The Counseling Psychologist, 19, 10-58.

Petrides, K. V., \& Furnham, A. (2003). Trait emotional intelligence: Behavioral validation in two studies of emotional recognition and reactivity to mood induction. European Journal of Personality, 17, 29-57. 
Pfeiffer, S. I. (2001). Emotional intelligence: Popular but elusive construct. Roeper Review, 23, $138-142$.

Poyrazli, S., Arbona, C., Bullington, R., \& Pisecco, S. (2001). Adjustment issues of Turkish college students studying in the United States. College Student Journal, 35, 52-62.

Robertson, M., Line, R., Jones, S., \& Thomas, S. (2000). International students, learning environments and perceptions: A case study using the Delphi technique. Higher Education \& Development, 19, 89-102.

Rozell, E. J., Pettijohn, C. F., \& Parker, R. S. (2002). An empirical evaluation of emotional intelligence: The impact on management development. Journal of Management Development, 21, 272-289.

Salovey, P., \& Mayer, J. D. (1990). Emotional intelligence. Imagination, Cognition and Personality, 9, $185-211$.

Schutte, N., Malouff, J., Hall, E., Haggerty, D., Cooper, J., \& Golden, D. et al. (1998). Development and validation of a measure of emotional intelligence. Personality and Individual Differences, 25, 167-177.

Scollon, C. N., Diener, E., Oishi, S., \& Biswas-Diener, R. (2004). Emotions across cultures and methods. Journal of Cross-cultural Psychology, 35, 304-326.

Shipper, F., Kincaid, J., Rotondo, D. M., \& Hoffman, R. C. (2003). A cross-cultural exploratory study of the linkage between emotional intelligence and managerial effectiveness. The International Journal of Organizational Analysis, 11, 171-191.

Sills, S. J., \& Song, C. (2002). Innovations in survey research: An application of web-based surveys. Social Science Computer Review, 20, 22-30.

Sitter, V. L. (2005). The effects of a leader's emotional intelligence on employee's trust in their leader and employee organizational citizenship behaviors. Dissertation Abstracts International, 65(9-A), 3467.

Slaski, M., \& Cartwright, S. (2002). Health, performance and emotional intelligence: An exploratory study of retail managers. Stress and Health, 18, 63-68.

Sternberg, R. J. (2001). Measuring the intelligence of an idea: How intelligent is the idea of emotional intelligence? In J. Ciarrochi, J. P. Forgas, \& J. D. Mayer (Eds.), Emotional intelligence in everyday life: A scientific inquiry (pp. 187-194). Philadelphia, PA: Psychology Press.

Tabachnick, B. G., \& Fidell, L. S. (1996). Using multivariate statistics (3rd ed.). New York: HarperCollins

Tett, R. P., Fox, K. E., \& Wang, A. (2005). Development and validation of a self-report measure of emotional intelligence as a multidimensional trait domain. Personality and Social Psychological Bulletin, 31, 859-888.

Thorndike, E.L. (1920). Intelligence and its uses. Harper's Magazine, 140, 227-235.

Tsaousis, I., \& Nikolaou, I. (2005). Exploring the relationship of emotional intelligence with physical and psychological health functioning. Stress and Health, 21, 77-86.

Van der Zee, K., Thijs, M., \& Schakel, L. (2002). The relationship of emotional intelligence with academic intelligence and the Big Five. European Journal of Personality, 16, 103-125.

Van Rooy, D. L., Alonso, A., \& Viswesvaran, C. (2005). Group differences in emotional intelligence scores: Theoretical and practical implications. Personality and Individual Differences, 38, 689-700.

Wong, C., \& Law, K. S. (2002). The effects of leader and follower emotional intelligence on performance and attitude: An exploratory study. The Leadership Quarterly, 13, 243-274.

Yoon, E., \& Portman, T. A. (2004). Critical issues of literature on counseling international students. Journal of Multicultural Counseling and Development, 32, 33-44. 